\title{
Avaliações do lúmen da artéria reparada em lesões do antebraço usando o teste de Allen, Doppler portátil e ultrassonografia com Doppler*
}

\section{Evaluations of Repaired Artery Patency in Forearm Wounds Using the Allen Test, Handheld Doppler and Doppler Ultrasonography}

\author{
Amanda Favaro Cagnolati ${ }^{10}$ Fernanda Ruiz Andrade ${ }^{1}$ Sara Dadona Correia Serrano ${ }^{2}$ \\ Claudio Henrique Barbieri ${ }^{3}$ Nilton Mazzer ${ }^{3}$ Marcello Henrique Nogueira-Barbosa ${ }^{4}$
}

\footnotetext{
${ }^{1}$ Residência Médica em Cirurgia de Mão, Departamento de Ortopedia e Anestesiologia, Faculdade de Medicina de Ribeirão Preto, Universidade de São Paulo, Ribeirão Preto, SP, Brasil

2 Programa de Cirurgia de Mão, Departamento de Ortopedia e Anestesiologia, Faculdade de Medicina de Ribeirão Preto, Universidade de São Paulo, Ribeirão Preto, SP, Brasil

3 Programa de Cirurgia de Mão, Departamento de Ortopedia e Anestesiologia, Faculdade de Medicina de Ribeirão Preto, Universidade de São Paulo, Ribeirão Preto, SP, Brasil

${ }^{4}$ Divisão de Radiologia, Departamento de Imagens Médicas, Hematologia e Oncologia Clínica, Faculdade de Medicina de Ribeirão Preto, Universidade de São Paulo, Ribeirão Preto, SP, Brasil
}

Endereço para correspondência Amanda Favaro Cagnolati, Departamento de Ortopedia e Anestesiologia, Faculdade de Medicina de Ribeirão Preto, Universidade de São Paulo, Campus Universitário., Ribeirão Preto, SP, Brasil (e-mail: amandafavaro@terra.com.br).

Rev Bras Ortop 2022;57(3):455-461.

\section{Resumo}

Palavras-chave

- artéria ulnar

- lesão arterial

- doppler

- lúmen arterial

- reparo arterial
Objetivo O objetivo deste estudo foi avaliar a taxa de perviedade pós-operatória de lesões arteriais do antebraço secundárias a traumatismo penetrante. As lesões foram submetidas a reparo primário e examinadas com o teste de Allen e um dispositivo Doppler portátil; posteriormente, os resultados foram confirmados à ultrassonografia com Doppler.

Métodos Dezoito pacientes foram incluídos, com um total de 19 lesões arteriais, 14 lesões ulnares e 5 lesões radiais; um paciente tinha lesões em ambos os antebraços. Todos os pacientes foram submetidos à cirurgia e três avaliações clínicas: o teste de Allen e a avaliação do fluxo sanguíneo arterial com um dispositivo portátil de Doppler na $4^{\mathrm{a}} \mathrm{e} 16^{\mathrm{a}}$ semanas após a cirurgia e ultrassonografia com Doppler 12 semanas após o procedimento.

Resultados Na primeira avaliação clínica, 77\% dos pacientes apresentavam perviedade segundo o teste de Allen e $72 \%$ apresentavam som pulsátil identificado pelo Doppler portátil. Na segunda avaliação, 61\% dos pacientes apresentaram perviedade com base no teste de Allen e a taxa de som pulsátil ao Doppler portátil foi de $72 \%$,
Estudo desenvolvido no Departamento de Ortopedia e Anestesiologia da Faculdade de Medicina de Ribeirão Preto, Universidade de São Paulo, Ribeirão Preto, SP, Brasil. recebido

10 de Julho de 2020

aceito

03 de Novembro de 2020

Publicado on-line

Agosto 13, 2021
DOI https://doi.org/

$10.1055 / \mathrm{s}-0041-1729574$. ISSN 0102-3616. (c) 2021. Sociedade Brasileira de Ortopedia e Traumatologia. All rights reserved.

This is an open access article published by Thieme under the terms of the Creative Commons Attribution-NonDerivative-NonCommercial-License, permitting copying and reproduction so long as the original work is given appropriate credit. Contents may not be used for commercial purposes, or adapted, remixed, transformed or built upon. (https://creativecommons.org/ licenses/by-nc-nd/4.0/)

Thieme Revinter Publicações Ltda., Rua do Matoso 170, Rio de Janeiro, RJ, CEP 20270-135, Brazil 


\begin{abstract}
Keywords

- ulnar artery

- arterial injury

- doppler

- arterial patency

- arterial repair

Objective The purpose of the present study was to evaluate the rate of patency in the postoperative period of arterial injuries of the forearm secondary to penetrating trauma. The injuries were subjected to primary repair and examined with the Allen test and a handheld Doppler device, and the results were later confirmed with Doppler ultrasonography.

Methods Eighteen patients were included, with a total of 19 arterial lesions, 14 ulnar lesions, and 5 radial lesions; one patient had lesions on both forearms. All patients underwent surgery and three clinical evaluations: the Allen test and assessment of arterial blood flow by a handheld Doppler device at 4 and 16 weeks after surgery and Doppler ultrasonography performed at 12 weeks after surgery.

Results At the first clinical evaluation, $77 \%$ of the patients had patency based on the Allen test, and $72 \%$ had a pulsatile sound identified by the handheld Doppler device. In the second evaluation, $61 \%$ of the patients had patency based on the Allen test, and the rate of pulsatile sound by the handheld Doppler device was $72 \%$, similar to that observed 2 months earlier. Based on the Doppler ultrasonography evaluation ( 12 weeks after surgery), the success rate for arteriorrhaphy was $88 \%$. Regarding the final patency (Doppler ultrasonography evaluation) and trauma mechanism, all patients with penetrating trauma had patent arteries.

Conclusion We conclude that clinical evaluation using a handheld Doppler device and the Allen test is reliable when a patent artery can be palpated. However, if a patent artery cannot be located during a clinical examination, ultrasonography may be required.
\end{abstract}

semelhante à observada 2 meses antes. À ultrassonografia com Doppler (cerca de 12 semanas após a cirurgia), a taxa de sucesso da arteriorrafia foi de $88 \%$. Em relação à perviedade final (avaliação por ultrassonografia com Doppler) e mecanismo de trauma, todos os pacientes com traumatismo penetrante apresentavam artérias pérvias.

Conclusão Concluímos que a avaliação clínica com um dispositivo Doppler portátil e o teste de Allen é confiável caso a artéria pérvia possa ser palpada. No entanto, a ultrassonografia pode ser necessária em caso de impossibilidade de localização de uma artéria pérvia durante o exame clínico.

\section{Introdução}

A lesão arterial do antebraço é responsável por $50 \%$ das lesões vasculares periféricas. ${ }^{1,2}$ Essas lesões são comumente associadas a traumatismos penetrantes ou contusos/penetrantes. Esse tipo de lesão é, em sua maioria, observado na população jovem do sexo masculino e é causado por lesão com vidro. ${ }^{3,4}$

De modo geral, essas lesões são acompanhadas por danos em estruturas musculotendíneas. Lesões ortopédicas e neurológicas geralmente são provocadas por traumatismo contuso. ${ }^{5,6}$

O diagnóstico de lesão arterial pode ser estabelecido à avaliação clínica; os sintomas incluem hemorragia, isquemia e déficits neurológicos associados devido à proximidade dos nervos às artérias. Exames complementares de imagem são indicados quando os sinais clínicos não podem confirmar a existência ou localização de uma lesão arterial. ${ }^{5,6}$

Exames clínicos, como palpação do pulso arterial, teste de Allen e Doppler portátil, podem ser usados para avaliação da perviedade arterial. A ultrassonografia com Doppler pode ser usada para avaliação como técnica de diagnóstico por imagem. ${ }^{5}$

No teste de Allen, o examinador oclui as artérias radial e ulnar por compressão digital e pede ao paciente que abra e feche a mão para drenar o sangue do arco palmar. $O$ paciente então relaxa a mão e o examinador para de comprimir a artéria a ser avaliada, observando o tempo necessário para normalização da cor da palma da mão. O teste é considerado positivo quando a artéria está pérvia, apresentando retorno à cor normal em menos de 6 segundos. ${ }^{1}$

A ultrassonografia com Doppler tem precisão de cerca de 98\% no diagnóstico de obstrução arterial e é considerada um exame não invasivo e de baixo custo. É mais específica do que sensível, além de demorada e dependente do examinador. ${ }^{4}$

Uma complicação associada ao reparo é a trombose do vaso, que leva ao insucesso do tratamento. A taxa de perviedade após o reparo da artéria radial ou da artéria ulnar com técnicas microcirúrgicas varia de cerca de 46 a $84 \%{ }^{4}$ As maiores taxas de sucesso são observadas nas lesões reparadas em até 
36 horas, causadas por objetos pontiagudos (não por avulsão ou laceração), e na artéria radial. ${ }^{4}$

Devido ao risco de trombose já discutido nos reparos arteriais, decidimos realizar exames clínicos e diagnósticos (ultrassonografia com Doppler) para a avaliação de pacientes submetidos à cirurgia de emergência para verificar a perviedade arterial e propor a avaliação clínica como alternativa à ultrassonografia com Doppler.

\section{Material e Métodos}

Pacientes com lesão da zona volar $\mathrm{V}$ do antebraço associada à lesão arterial foram submetidos ao reparo primário da lesão e acompanhados com avaliação clínica e ultrassonográfica no Hospital das Clínicas da Faculdade de Medicina de Ribeirão Preto entre maio de 2018 e setembro de 2019.

Os pacientes submetidos à ligadura da artéria danificada ou com necessidade de interposição do enxerto para reparo arterial foram excluídos.

Dos 33 pacientes selecionados, 15 não completaram o acompanhamento no ambulatório, ou seja, não retornaram para as consultas e devidas avaliações. Obtivemos todas as informações necessárias para o estudo de 18 pacientes, em sua maioria (72\%) homens jovens, com idade média de 30 anos (variação de 16-60 anos) e sem comorbidades.

Os pacientes retornaram para uma consulta de acompanhamento em 1 semana para avaliação do curativo e em 4 semanas para avaliação da amplitude de movimento (caso apresentassem lesão tendínea associada) e do fluxo arterial por meio do teste de Allen e Doppler portátil (-Figs. 1 e 2). Cerca de 12 semanas após a lesão, os pacientes foram avaliados à ultrassonografia com Doppler e, aproximadamente 14 a 16 semanas após a lesão, foram reavaliados pelo teste de Allen e exame com Doppler portátil.

O mecanismo de trauma mais frequente foi o traumatismo penetrante, responsável por cerca de $78 \%$ dos casos; em $22 \%$ dos casos, o mecanismo foi o traumatismo contuso/penetrante, como em acidentes de trabalho.

A sutura mais utilizada foi o polipropileno (88\%), enquanto o náilon foi utilizado em $12 \%$ dos casos, de acordo com a escolha do cirurgião.

Todos os reparos foram realizados com lupas, com aumento de até 3,5 vezes em 9 casos e de 4 a 6 vezes em 9 casos. Todas as cirurgias foram realizadas de maneira idêntica por dois cirurgiões que estavam no final do treinamento em cirurgia da mão e sob a tutela do autor sênior.

Setenta e sete por cento dos pacientes foram submetidos à arteriorrafia em até 120 horas (5 dias) após a lesão, com tempo médio de 82 horas (cerca de 3 dias após a lesão).

A análise estatística descritiva dos dados foi realizada. 0 teste $t$ de Student, o teste exato de Fisher ${ }^{7}$ e o teste de McNemar foram usados. Todas as análises estatísticas foram conduzidas com o software estatístico SAS 9.4 (SAS Institute Inc., Cary, NC, EUA)..$^{7-9}$

Este estudo foi aprovado pelo Comitê de Ética em Pesquisa em Seres Humanos e todos os participantes assinaram o termo de consentimento livre e esclarecido.

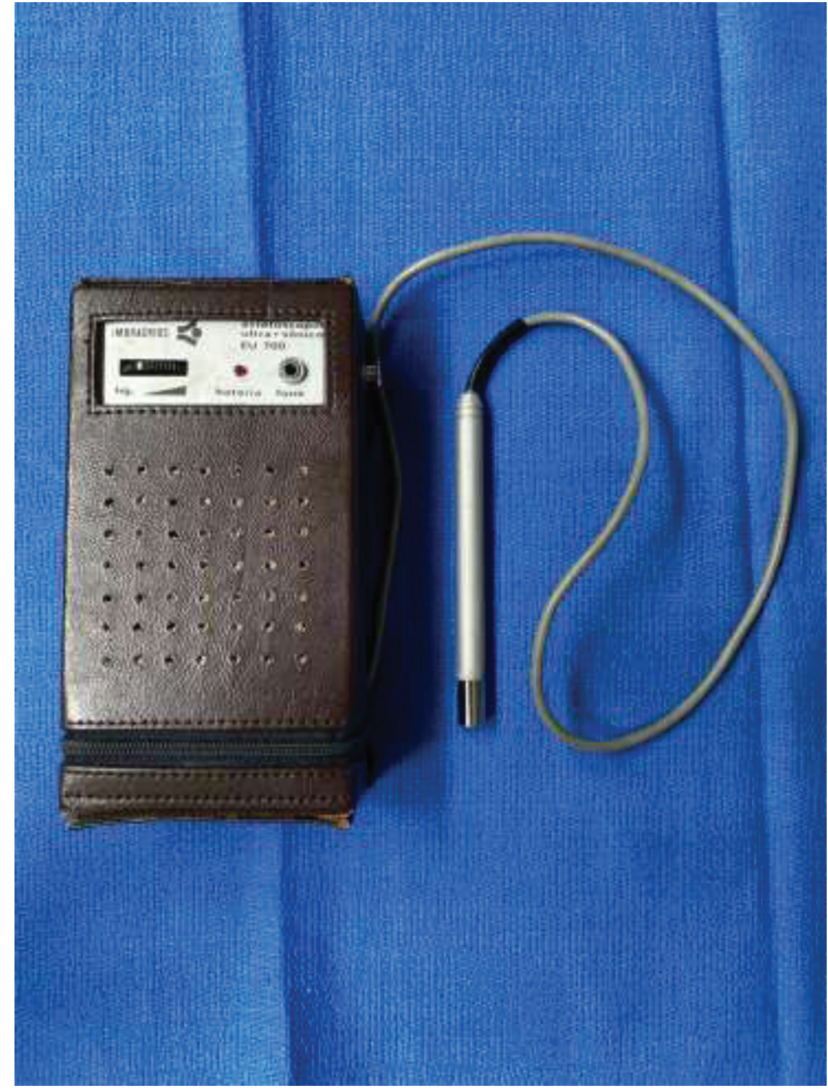

Fig. 1 Dispositivo Doppler portátil usado para avaliação dos pacientes.

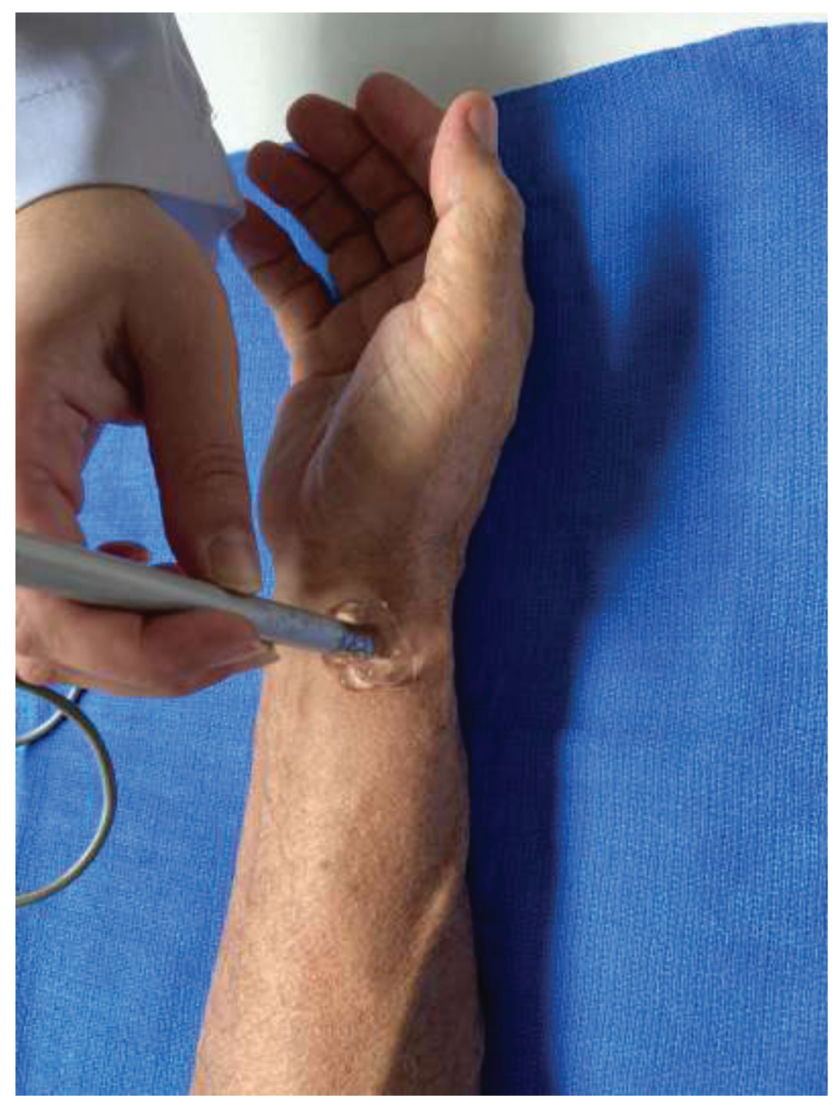

Fig. 2 Avaliação de um paciente com Doppler portátil. 
Tabela 1 Comparação do lúmen arterial determinado à ultrassonografia com Doppler em pacientes submetidos à arteriorrafia precoce ou tardia

\begin{tabular}{|l|l|l|l|}
\hline \multirow{2}{*}{ INTERVALO } & \multicolumn{2}{|l|}{ DOPPLER } & \multirow{2}{*}{ Total } \\
\cline { 2 - 3 } & $\begin{array}{l}\text { Lúmen } \\
\text { obstruído }\end{array}$ & $\begin{array}{l}\text { Lúmen } \\
\text { desobstruído }\end{array}$ & \\
\hline até $\mathbf{1 2 0}$ horas & 0 & 14 & 14 \\
& 0,00 & 77,78 & 77,78 \\
\hline$>\mathbf{1 2 0}$ horas & 2 & 2 & 4 \\
& 11,11 & 11,11 & 22,22 \\
\hline Total & 2 & 16 & 18 \\
& 11,11 & 88,89 & 100,00 \\
\hline
\end{tabular}

Teste exato de Fisher: valor de $p=0,0392$.

\section{Resultados}

Na primeira avaliação clínica (cerca de 4 semanas após a lesão), 77\% dos pacientes apresentaram perviedade com base no teste de Allen; o pulso foi identificado com Doppler portátil em $72 \%$ dos pacientes (-Figs. 1 e 2 ).

Na segunda avaliação clínica (cerca de 14 a 16 semanas após a lesão), 61\% dos pacientes apresentaram perviedade com base no teste de Allen e a taxa de identificação de pulso com Doppler portátil continuou em $72 \%$, como observado 2 meses antes.

A avaliação por ultrassonografia com Doppler (cerca de 12 semanas após a lesão) indicou uma taxa de sucesso da arteriorrafia de $88 \%$. Em relação à perviedade final (avaliação por ultrassonografia com Doppler) e ao mecanismo de trauma, todos os pacientes com traumatismo penetrante apresentavam artérias pérvias; a taxa de sucesso foi de 85\% em pacientes com traumatismo contuso/penetrante (valor de $p$ de 1,0 ).

Todos os pacientes submetidos à arteriorrafia com fio de náilon apresentavam artérias pérvias à avaliação final. Entre os pacientes submetidos à arteriorrafia com polipropileno, 87\% apresentaram perviedade à ultrassonografia com Doppler (valor de $p$ de 1,0).

Todas as cirurgias foram realizadas com uso de lupa de $3,5 \times$ ou $4 \times$ de aumento durante o reparo e não houve diferença entre os grupos (valor de $p$ de 1,0 ) ou entre os cirurgiões.

Todos os pacientes submetidos ao reparo em 5 dias apresentavam artérias pérvias à avaliação final. A taxa de perviedade em pacientes submetidos à arteriorrafia tardia foi de $50 \%$ (valor de $p$ de 0,03 ) (-Tabela 1). Todos os pacientes com resultados positivos no teste de Allen à primeira avaliação clínica (4 semanas) apresentaram artérias pérvias à avaliação ultrassonográfica final. Dos pacientes com resultados negativos ao teste de Allen (oclusão arterial) à primeira avaliação clínica, apenas $50 \%$ tinham artérias pérvias à avaliação final de ultrassonografia com Doppler (valor de $p$ de 0,03)(-Fig. 3). Na segunda avaliação clínica (14-16 semanas), a concordância do teste de Allen com a avaliação ultrassonográfica continuou em $100 \%$ nos pacientes com artérias pérvias com base no teste de Allen, mas a taxa de concordância diminuiu para $28 \%$ para pacientes com resultados negativos no teste de Allen (valor de $p$ de 0,13 ) (-Fig. 4).

O exame com Doppler portátil realizado durante a primeira avaliação clínica também mostrou $100 \%$ de concordância com a avaliação ultrassonográfica em pacientes com artérias pérvias e 40\% de concordância quando não houve localização

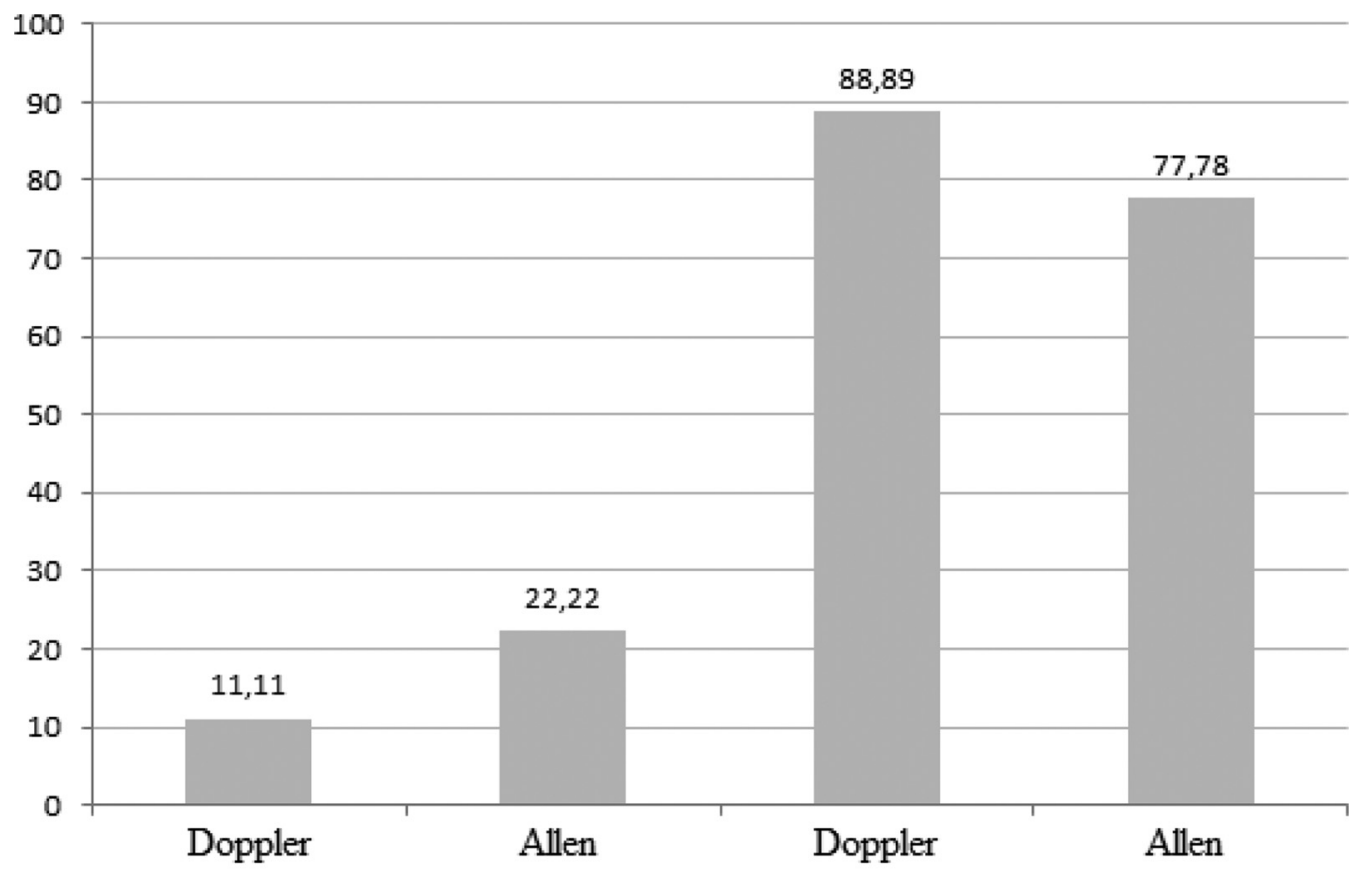

Artéria não pérvia

Artéria pérvia

Fig. 3 Comparação do primeiro teste de Allen com a última ultrassonografia. 


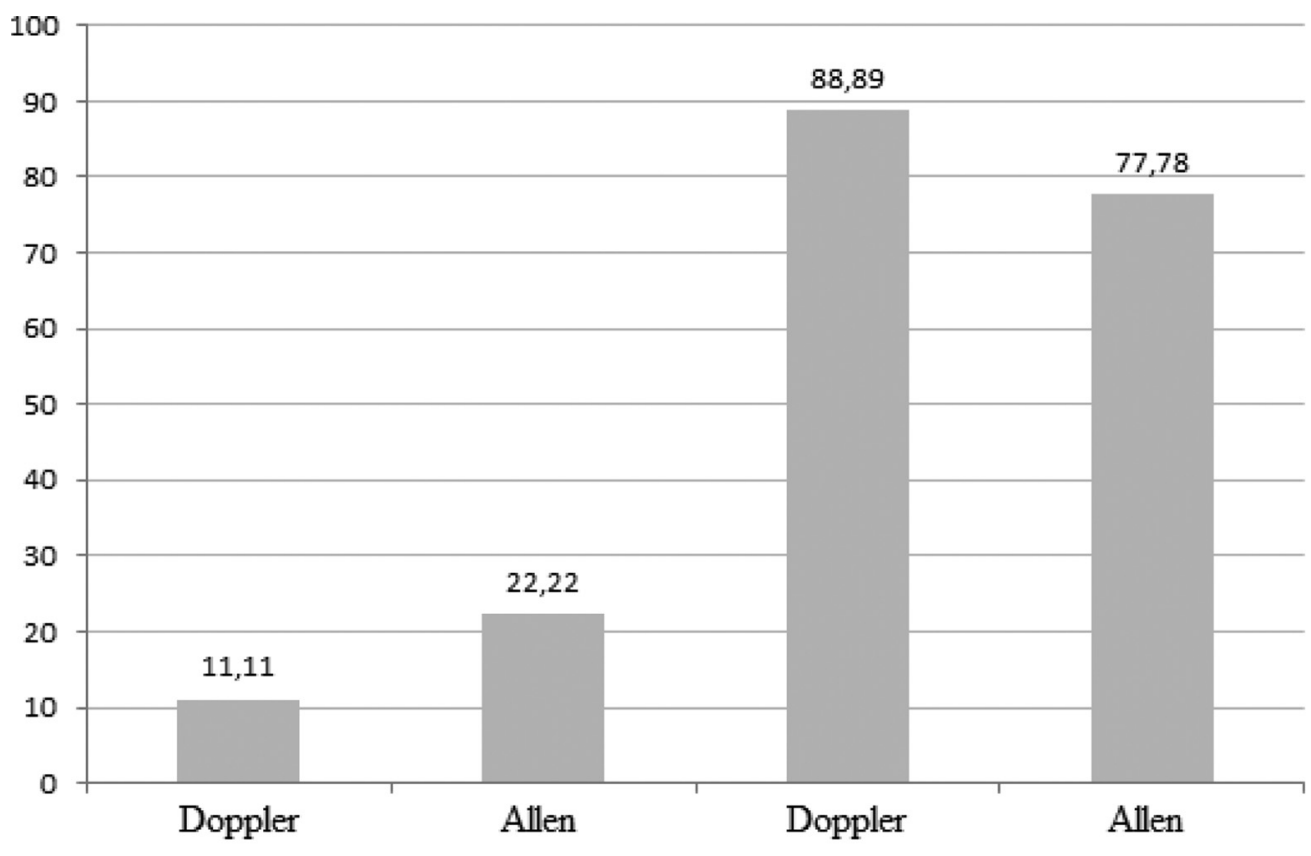

Artéria não pérvia

Artéria pérvia

Fig. 4 Comparação do segundo teste de Allen com última ultrassonografia.

do pulso (valor de $p$ de 0,06 ). Esses parâmetros foram mantidos na segunda avaliação clínica (-Fig. 5).

\section{Discussão}

O tratamento das lesões arteriais pode ser feito por ligadura, reparo primário (em lacerações com menos de $2 \mathrm{~cm}$ e boa mobilidade dos cotos para suturas sem tensão) ou reparo com interposição de enxerto (em lacerações com mais de $2 \mathrm{~cm})^{10,11}$

Em caso de lesão das duas artérias (radial e ulnar), mesmo que a mão esteja bem perfundida, o reparo arterial deve ser realizado para diminuir os sintomas de isquemia. ${ }^{1,4}$ Alguns estudos demonstraram que a perda de perfusão pela artéria

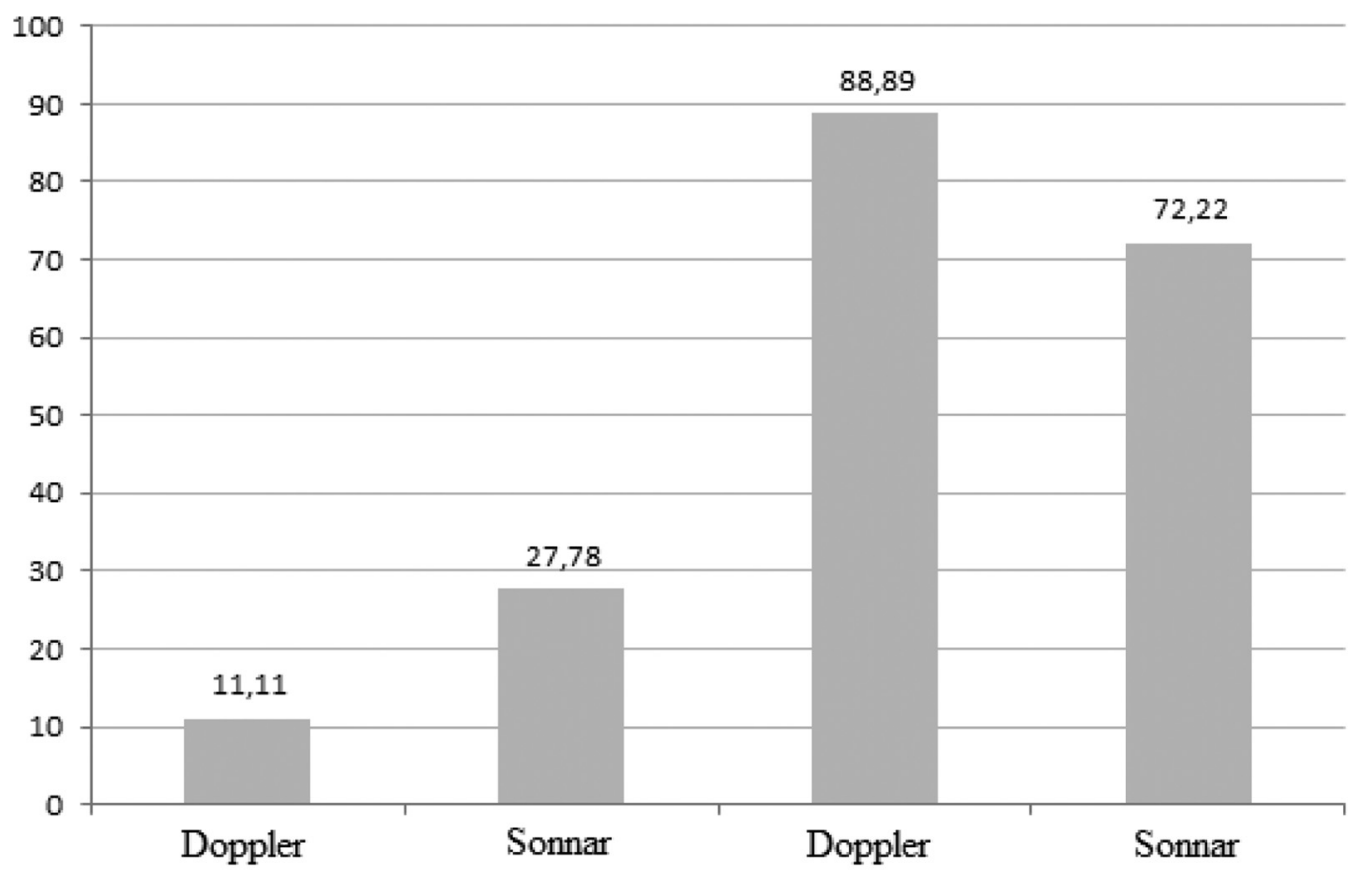

Artéria não pérvia

Artéria pérvia

Fig. 5 Comparação da primeira avaliação com Doppler portátil à última avaliação por ultrassonografia. 
radial ou ulnar para a mão pode causar intolerância ao frio, atrofia muscular e óssea e perda de força. ${ }^{12}$ Isso confirma a importância do reparo arterial e da perviedade mesmo em lesões de uma única artéria em uma mão bem perfundida.

Na presença de uma lesão neurológica associada, há um ganho importante na recuperação quando a perviedade da artéria é alcançada após o reparo arterial. De acordo com a literatura, $87 \%$ dos pacientes com lesão do nervo ulnar obtiveram recuperação neurológica boa, adequada ou excelente em caso de perviedade da artéria ulnar; a taxa de bons resultados diminuiu para $33 \%$ em pacientes com obliteração da artéria ulnar. ${ }^{5,12}$

As causas da trombose do vaso ainda não são bem compreendidas, mas acredita-se que a pressão retrógrada no arco palmar provoca turbulência vascular e diminui o fluxo na artéria reparada, levando à sua obstrução. ${ }^{13,14}$

Como a epidemiologia relatada por outros estudos, em nossa amostra, a maior prevalência de lesões arteriais no antebraço foi observada em pacientes jovens do sexo masculino.

A ultrassonografia com Doppler é uma técnica não invasiva, rápida e precisa para avaliação dos resultados do reparo vascular, além da taxa e das características do fluxo; em nosso estudo, a taxa de sucesso de demonstração de artérias pérvias à ultrassonografia com Doppler foi de $88 \%$, índice superior ao relatado na literatura, de até $84 \%$. ${ }^{2,4}$ A maior taxa de sucesso ocorreu em pacientes com trauma penetrante (100\%) e chegou a $85 \%$ nos casos de trauma penetrante/contuso.
Ao analisarmos a perviedade final em relação ao tipo de sutura utilizada, o maior índice de sucesso foi associado ao uso de náilon (100\%) em comparação a polipropileno (87\%); entretanto, como apenas duas artérias foram reparadas com náilon (dois procedimentos), esse achado tem pouca significância estatística (valor de $p$ de 1,0). Segundo a literatura, a taxa de perviedade é semelhante entre os tipos de suturas, com melhor biocompatibilidade associada ao polipropileno. ${ }^{15}$

Todos os reparos foram realizados com lupas cirúrgicas, e a literatura demonstra que o uso de técnicas microcirúrgicas aumenta a taxa de sucesso da arteriorrafia.

Em relação ao intervalo entre a lesão e o reparo, obtivemos uma taxa de perviedade de $100 \%$ nas artérias reparadas em 5 dias (-Fig. 6), com diminuição significativa para $50 \%$ quando o intervalo era superior a 5 dias e boa significância estatística para esses dados (valor de $p$ de 0,03 ). Tivemos uma alta taxa de sucesso mesmo quando o procedimento foi realizado depois de 36 horas, ao contrário do relatado na literatura. $^{4}$

Quanto às avaliações clínicas e ultrassonográficas, constatamos que tanto o teste de Allen quanto o Doppler portátil foram confiáveis para determinar a perviedade arterial ao indicarem resultados positivos (artéria pérvia), com alto valor preditivo positivo. No entanto, os resultados da ultrassonografia com Doppler não foram bem correlacionados aos resultados dos exames clínicos que mostram oclusão arterial (-Fig. 7), gerando muitos falso-negativos. O tamanho de nossa amostra foi pequeno, mas propomos que as avaliações clínicas são suficientes para avaliar o reparo vascular. ${ }^{4}$

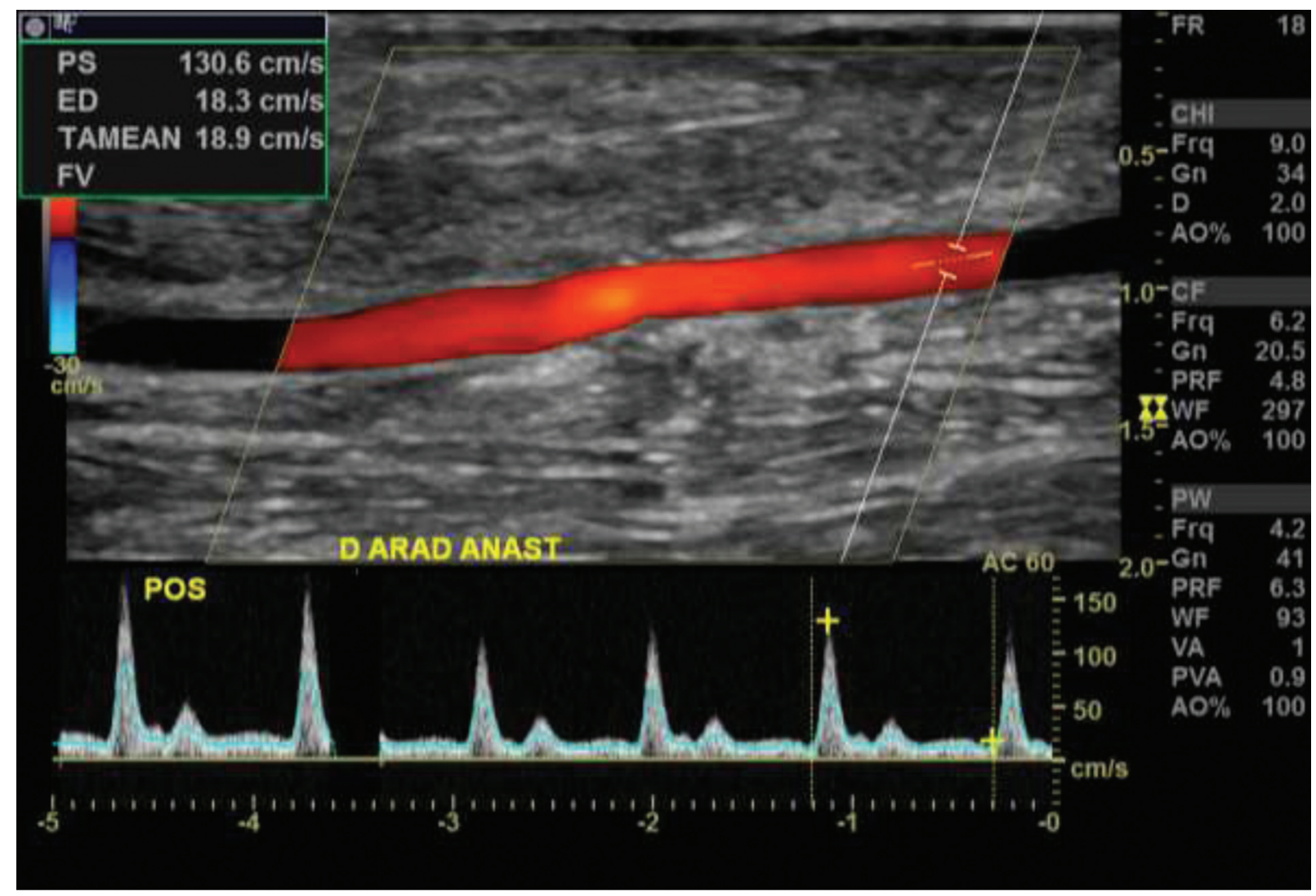

Fig. 6 A ultrassonografia com Doppler revela a artéria pérvia após a arteriorrafia. 


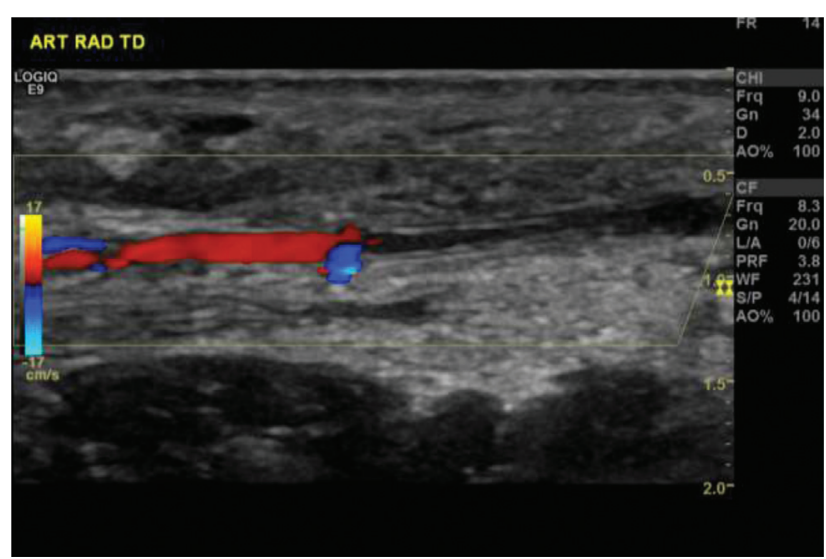

Fig. 7 A ultrassonografia com Doppler revela uma artéria obstruída.

Concluímos que a avaliação clínica com Doppler portátil e o teste de Allen é confiável ao mostrar uma artéria pérvia. No entanto, se a avaliação clínica revelar artérias não pérvias, o resultado deve ser confirmado por ultrassonografia.

\section{Suporte Financeiro}

Este trabalho teve suporte do Instituto de Pesquisa e Ensino Home, Brasília, DF, Brasil.

\section{Conflito de Interesses}

Os autores não têm conflito de interesses a declarar.

\section{Referências}

1 Thai JN, Pacheco JA, Margolis DS, et al. Evidence-based comprehensive approach to forearm arterial laceration. West J Emerg Med 2015;16(07):1127-1134

2 Lannau B, Bliley J, James IB, et al. Long-term patency of primary arterial repair and the modified cold intolerance symptom seve- rity questionnaire. Plast Reconstr Surg Glob Open 2015;3(11): e551

3 Andreev A, Kavrakov T, Karakolev J, Penkov P. Management of acute arterial trauma of the upper extremity. Eur J Vasc Surg 1992;6(06):593-598

4 Lebowitz C, Matzon JL. Arterial injury in the upper extremity: evaluation, strategies, and anticoagulation management. Hand Clin 2018;34(01):85-95

5 Keleş MK, Şimşek T, Polat V, Yosma E, Demir A. Evaluation of forearm arterial repairs: Functional outcomes related to arterial repair. Ulus Travma Acil Cerrahi Derg 2017;23(02):117-121

6 Franz RW, Goodwin RB, Hartman JF, Wright ML. Management of upper extremity arterial injuries at an urban level I trauma center. Ann Vasc Surg 2009;23(01):8-16

7 Fisher RA. The logic of inductive inference. J R Stat Soc 1935; 98:39-82

8 Pagano M, Gauvreau KPrincípios de bioestatística. Tradução de luiz sérgio de castro paiva. Revisão técnica de lúcia pereira barroso. São Paulo: Pioneira Thomson Learning; 2004

9 SAS Institute Inc. SAS/STAT® user's guide, version 9.4. Cary, NC: SAS Institute Inc; 2019

10 Gelberman RH, Blasingame JP, Fronek A, Dimick MP. Forearm arterial injuries. J Hand Surg Am 1979;4(05):401-408

11 Bornmyr S, Arner M, Svensson H. Laser Doppler imaging of finger skin blood flow in patients after microvascular repair of the ulnar artery at the wrist. J Hand Surg Br 1994;19(03):295-300

12 Rothkopf DM, Chu B, Gonzalez F, Borah G, Ashmead D 4th, Dunn R. Radial and ulnar artery repairs: assessing patency rates with color Doppler ultrasonographic imaging. J Hand Surg Am 1993;18(04): 626-628

13 Rasulic L, Cinara I, Samardzic M, et al. Nerve injuries of the upper extremity associated with vascular trauma-surgical treatment and outcome. Neurosurg Rev 2017;40(02):241-249

14 Nunley JA, Goldner RD, Koman LA, Gelberman R, Urbaniak JR. Arterial stump pressure: a determinant of arterial patency? J Hand Surg Am 1987;12(02):245-249

15 Chen LE, Seaber AV, Urbaniak JR. Comparison of 10-0 polypropylene and 10-0 nylon sutures in rat arterial anastomosis. Microsurgery 1993;14(05):328-333 